\title{
Gradient Nanomechanics: Applications to Deformation, Fracture, and Diffusion in Nanopolycrystals
}

\begin{abstract}
E.C. AIFANTIS
The term "gradient nanomechanics" is used here to designate a generalized continuum mechanics framework accounting for "bulk-surface" interactions in the form of extra gradient terms that enter in the balance laws or the evolution equations of the relevant constitutive variables that govern behavior at the nanoscale. In the case of nanopolycrystals, the grain boundaries may be viewed either as sources/sinks of "effective" mass and internal force or as a separate phase, interacting with the bulk phase that it surrounds, and supporting its own fields, balance laws, and constitutive equations reflecting this interaction. In either view, a further common assumption introduced is that the constitutive interaction between bulk and "interface" phases enters in the form of higher order gradient terms, independently of the details of the underlying physical mechanisms that bring these terms about. The effectiveness of the approach is shown by considering certain benchmark problems for nanoelasticity, nanoplasticity, and nanodiffusion for which standard continuum mechanics theory fails to model the observed behavior. Its implications to interpreting size-dependent stress-strain curves for nanopolycrystals with varying grain size are also discussed.
\end{abstract}

DOI: $10.1007 / \mathrm{s} 11661-011-0725-9$

(C) The Minerals, Metals \& Materials Society and ASM International 2011

\section{INTRODUCTION}

ConTINUUM mechanics was used as an effective tool to model material behavior and processes across a variety of scales ranging from macroscopic (construction/manufacturing industries) and microscopic (optoelectronics/micro-electro-mechanical systems or MEMS technologies) to planetary (earthquakes/tsunamis) and cosmological (star formation/galaxy clustering) ones. Its applicability to the nanoscale has not been explored systematically since this field has emerged only recently and is usually dominated by computer simulations. Moreover, the key submicroscopic mechanisms that such a continuum description should be based upon are not clear. A first goal of this article is to illustrate how the basic structure of an earlier proposal of the author for media with microstructure can be extended to describe deformation and transport processes at the nanoscale. Continuum models for nanoelasticity, nanoplasticity, and nanodiffusion are derived within such a generalized framework. A second objective is to show the effectiveness of these models in describing nanoscale phenomena observed in benchmark configurations that may not be captured by classical continuum theory. They are concerned with the dependence of the effective elastic modulus on the grain size in elastic bicrystals, the inverse Hall-Petch (H-P) effect in nanopolycrystals, and the curvature of concentration-depth profiles observed

E.C. AIFANTIS, Professor, is with the Laboratory of Mechanics and Materials, Polytechnic School, Aristotle University of Thessaloniki, GR-54124 Thessaloniki, Greece. Contact e-mail: mom@mom. gen.auth.gr

Manuscript submitted November 8, 2010.

Article published online June 4, 2011 in nanophase materials. A third objective is to illustrate with two characteristic examples how dislocation theory and fracture mechanics can be revisited with gradient elasticity to dispense with classical singularities and provide information on dislocation core and crack tip effects, which become important at the nanoscale. A final objective is to show how a simplified approximate treatment of the gradient terms can efficiently capture size-dependent stress-strain curves of deforming nanopolycrystals with varying grain size under different temperature and strain rate conditions.

The basic premise that the proposed nanomechanics framework builds upon is the explicit recognition of the critical role of the "surface to volume" ratio in revising the standard continuum mechanics model. This is done by introducing extra terms in the usual balance laws (mass, momentum) or the evolution equations of the basic variables (plastic strain, defect densities) and, then, by making appropriate constitutive assumptions for these extra terms. The constitutive assumptions for the extra terms are motivated directly by the theory of gradient elasticity for the case of elastic deformation (nanoelasticity), gradient plasticity for the case of plastic flow (nanoplasticity), and the theory of double diffusivity for the case of diffusion (nanodiffusion). It turns out that incorporation of gradient-dependent constitutive equations into the aforementioned extended "bulksurface" continuum framework results in an effective and robust methodology for interpreting deformation, flow, and diffusion problems at the nanoscale. In this connection, it is emphasized that a large number of articles addressing material behavior at the nanoscale are usually based either on a direct and straightforward adoption of corresponding models at macro- and micro-scales or on elaborate but also straightforward 
computer simulations involving ab-initio and molecular dynamics (MD) procedures based on empirical potentials. The direct adaptation of macro- and micro-scale models to consider material behavior at the nanoscale is questionable, since there is ample experimental evidence of strong size effects observed as a characteristic material or specimen dimension crosses over from the micron to the nano regime; and these effects cannot be captured by such standard models without proper modification. On the other hand, numerical simulations are always restricted by the large computer times required and the unrealistic (as opposed to experimentally imposed) strain rates assumed for such computations. The point of view advanced in the present approach seems to be a reasonable compromise. It maintains the basic continuum mechanics methodology and structure but endows it with an internal length parameter quantifying the effect of higher order gradients on the form of the extra terms modeling the bulksurface interaction, independently of the details of the underlying submicroscopic mechanisms that bring these terms about (i.e., the terms modeling the exchange of "effective mass" and "effective momentum" between "bulk" and internal or external "surface" points). This point of view is motivated by earlier proposals of the author for a continuum with microstructure, ${ }^{[1-3]}$ sketched in an effort to model self-diffusion and plastic instabilities in solids. The carriers of effective "mass" and "momentum" in the case of self-diffusion are the vacancies, and the associated surface irregularities resulting by their internal motion are "smoothed out" by appropriate effective boundary conditions for their concentration and flux. In the case of plasticity, the internal carriers of mass and momentum are dislocations and related structural defects and, therefore, dislocation mechanics should be used to motivate the appropriate constitutive equations.

The presentation is divided into four parts. In the first part (Section II), the basic structure of the bulk-surface interaction nanomechanics framework is outlined for the case of nanoelasticity, nanoplasticity, and nanodiffusion. In the second part (Section III), we use such nanoelasticity, nanoplasticity, and nanodiffusion models to discuss certain benchmark nanoscale configurations. We derive results for the grain size dependence of effective elastic moduli, H-P type normal and abnormal behavior in nanopolycrystals, and the curvature observed experimentally in concentration-penetration diffusion profiles in nanophase materials. In the third part (Section IV), we present two representative examples from dislocation theory and fracture mechanics where elastic singularities are eliminated and the details of such nonsingular stress and strain distributions near dislocation cores and crack tips can be obtained in conjunction with their use in related nanoscale applications. Finally, in the fourth part (Section V), size-dependent stress-strain curves for nanopolycrystals of varying grain size are obtained and compared with recent experimental data. The effects of strain rate and temperature are also discussed in good agreement with observed behavior. In this discussion, the effect of strain gradients is accounted for by an oversimplified but robust nanoscopic dimensional argument.

\section{GRADIENT CONTINUUM NANOMECHANICS FRAMEWORK}

An extended generalized continuum mechanics framework is outlined here for addressing the mechanical response of nanocrystalline (NC) and ultrafine grain (UFG) polycrystals. This extension is based on generalizing the standard continuum mechanics structure by introducing extra terms modeling the "interaction" between bulk and (external or internal) surface points, as well as appropriate constitutive equations for these terms. An alternative physical concept that can be used to achieve such a generalization is to view NC and UFG polycrystals as a mixture of "bulk" and "grain boundary" phases. The two phases can interact mechanically by exchanging mass and momentum, but the overall "composite" should obey the standard balance laws of continuum mechanics with each phase obeying its own constitutive equations. We present this discussion, for convenience, separately for elastic and plastic deformations, and separately for diffusion at the nanoscale. The resulting governing differential model equations are proposed to be used in connection with the determination of the mechanical and diffusion response of polycrystals at the submicron and nano regimes. We will refer to these models as nanoelastiticy, nanoplasticity, and nanodiffusion, respectively.

\section{A. Nanoelasticity}

\section{Bulk/surface approach}

Within this approach, the standard equilibrium equation $\operatorname{div} \boldsymbol{\sigma}=0$ is generalized to include an additional internal body force $\hat{\mathbf{f}}$ representing the exchange of momentum between bulk and surface points. Then, the balance law of linear momentum in the absence of inertia effects reads

$$
\operatorname{div} \boldsymbol{\sigma}^{b}=\hat{\mathbf{f}}
$$

or, in indicial notation, $\sigma_{i j, k}=\hat{f}_{i}$, where $\boldsymbol{\sigma}^{b}$ is the usual second-order stress tensor of the bulk material and $\hat{\mathbf{f}}$ is an internal-like force modeling the momentum exchange between the bulk and surface points. It is further assumed that $\hat{\mathbf{f}}$ is determined by a third-order stress or "hyperstress" $\mathcal{M}$ of third order, which, in turn, may be expressed as the gradient of the secondorder stress $\mathbf{S}$ or extra stress modeling the bulk-surface interaction. It follows that

$$
\hat{\mathbf{f}}=\operatorname{div} \operatorname{div} \mathcal{M} ; \mathcal{M}=\nabla \mathbf{S}
$$

or, in indicial notation, $\hat{f}_{i}=\mathcal{M}_{i j k, j k} ; \mathcal{M}_{i j k, j k}=\mathrm{S}_{i j, k}$, where the index summation convention is adopted.

The simplest possible constitutive assumption for the extra stress $\mathbf{S}$ is to take it proportional to the bulk stress $\boldsymbol{\sigma}^{b}$; i.e.,

$$
\mathbf{S}=c \boldsymbol{\sigma}^{b}
$$

and then Eq. [1] can be written as

$$
\operatorname{div} \boldsymbol{\sigma}=0 ; \boldsymbol{\sigma}=\boldsymbol{\sigma}^{b}-c \nabla^{2} \boldsymbol{\sigma}^{b}
$$

If for $\boldsymbol{\sigma}^{b}$ we adopt the usual Hooke's law of classical elasticity, i.e., $\boldsymbol{\sigma}^{b}=\lambda(\operatorname{tr} \boldsymbol{\varepsilon}) \mathbf{1}+2 \mathrm{G} \boldsymbol{\varepsilon}$, where $(\lambda, G)$ denote 
the Lamé constants; it follows that the total stress $\boldsymbol{\sigma}$, including both the usual stress corresponding to the bulk and its interaction with the surface, is determined by the equations

$$
\operatorname{div} \boldsymbol{\sigma}=0 ; \quad \boldsymbol{\sigma}=\lambda(\operatorname{tr} \boldsymbol{\varepsilon}) \mathbf{1}+2 G \boldsymbol{\varepsilon}-c \nabla^{2}[\lambda(\operatorname{tr} \boldsymbol{\varepsilon}) \mathbf{1}+2 G \boldsymbol{\varepsilon}]
$$

i.e., the equations of gradient elasticity.

\section{Mixture approach}

As discussed earlier, a two-phase composite material model may also be adopted for deriving differential equations for the deformation field in a nanograined polycrystal. According to this assertion, it may be assumed that bulk and grain boundary phases occupy the same material point but locally interact via an internal body force $\hat{\mathbf{f}}$. The differential equations of equilibrium are then expressed in the form ${ }^{[4]}$

$$
\operatorname{div} \boldsymbol{\sigma}_{1}=\hat{\mathbf{f}} ; \quad \operatorname{div} \boldsymbol{\sigma}_{2}=-\hat{\mathbf{f}} \quad \Rightarrow \quad \operatorname{div} \boldsymbol{\sigma}=0
$$

with $\left(\boldsymbol{\sigma}_{1}, \boldsymbol{\sigma}_{2}\right)$ denoting the partial stress tensors for each individual phase and $\boldsymbol{\sigma}=\sigma_{1}+\sigma_{2}$ being the stress tensor for the nanostructured material considered as a whole. By assuming that each phase obeys Hooke's law and that the interaction force is proportional to the difference of the individual displacements, we have the relationships (for $k=1,2$ )

$$
\boldsymbol{\sigma}_{k}=\lambda_{k}\left(\operatorname{tr} \boldsymbol{\varepsilon}_{k}\right) 1+2 G_{k} \boldsymbol{\varepsilon}_{k} ; \quad \hat{\mathbf{f}}=\alpha\left(\mathbf{u}_{1}-\mathbf{u}_{2}\right)
$$

where $\left(\lambda_{k}, G_{k}\right)$ and $\alpha$ are phenomenological coefficients, $\mathbf{1}$ is the identity tensor, while (div, $\nabla$ ) denote the divergence and gradient operators, respectively. The $\nabla$ operator enters in the definition of the strain tensor $\varepsilon$ in terms of the diplacement vector $\mathbf{u}$, i.e. $\boldsymbol{\varepsilon}=(1 / 2)$ $\left[\nabla \mathbf{u}+(\nabla \mathbf{u})^{\mathrm{T}}\right]$, with ${ }^{\mathrm{T}}$ denoting transposition. Uncoupling, leads to the following differential equation for the total displacement $\mathbf{u}=(1 / 2)\left(\mathbf{u}_{1}+\mathbf{u}_{2}\right)$ associated with the nanostructured material

$$
G \nabla^{2} \mathbf{u}+(\lambda+G) \operatorname{div} \mathbf{u}-c \nabla^{2}\left[G \nabla^{2} \mathbf{u}+(\lambda+G) \operatorname{div} \mathbf{u}\right]=0
$$

where the coefficients $(\lambda, G, c)$ are related explicitly to those appearing in Eq. [7], which, in turn, should satisfy certain special conditions in order that only one gradient coefficient $c$ appears in the final form of the governing differential equations for the total displacement. As a direct consequence of Eq. [8], and within an additive divergence-free term, the following gradient modification of Hooke's law may be suggested to model elastic deformation at the nanoscale (nanoelasticity):

$$
\boldsymbol{\sigma}=\lambda(\operatorname{tr} \boldsymbol{\varepsilon}) \mathbf{1}+2 G \boldsymbol{\varepsilon}-c \nabla^{2}[\lambda(\operatorname{tr} \boldsymbol{\varepsilon}) \mathbf{1}+2 G \boldsymbol{\varepsilon}]
$$

where the total strain $\boldsymbol{\varepsilon}$ is defined in the usual way in terms of the overall displacement $\mathbf{u}$ (see above).

\section{B. Nanoplasticity}

\section{Bulk/surface approach}

To derive modified equations for plasticity, along similar lines as for elasticity, we start with the one-dimensional configuration of simple shear. In this case, the "equivalent" or "effective" shear stress, which is used as the basic quantity for deriving constitutive plasticity equations in three dimensions, may be identified with the bulk shear stress driving plastic flow on the assumed representative slip plane. Thus, for simple shear conditions, we have

$$
\partial_{x} \tau^{b}=\hat{f}
$$

where $\tau^{b}$ is the bulk shear stress in a direction perpendicular to the $x$-coordinate (i.e. the coordinate perpendicular to the slip direction) and $\hat{f}$ is the onedimensional scalar counterpart of the exchange of momentum force between bulk and surface points. As in the elastic case, it is assumed that the scalar $\hat{f}$ is given by the spatial derivative of another scalar field $\mathcal{M}$, which, in turn, is given by the spatial derivative of another scalar field $\mathrm{S}$; i.e.,

$$
\hat{f}=\partial_{x} \mathcal{M} ; \quad \mathcal{M}=\partial_{x} \mathrm{~S}
$$

Unlike in the elastic case where the interaction of bulk and surface points can be expressed equivalently either in terms of stress or in terms of strain, in the case of plasticity, it is assumed here that the shear strain $\gamma$ determines the bulk-surface interaction. In other words, a linear relationship of the form

$$
\mathrm{S}=c \gamma
$$

is proposed, with $c$ denoting a phenomenological coefficient (not to be confused with the one used in Eq. [8]). Thus, in view of Eq. [12], Eq. [11] can be written as

$$
\partial_{x} \tau=0 ; \quad \tau=\kappa(\gamma)-c \partial_{x x} \gamma
$$

where the standard isotropic hardening relation $\tau^{\mathrm{b}}=\kappa(\gamma)$ was assumed for the bulk stress. The threedimensional generalization of Eq. [13] reads

$$
\operatorname{div} \boldsymbol{\sigma}=0 ; \quad \tau=\kappa(\gamma)-c \nabla^{2} \gamma
$$

where $\boldsymbol{\sigma}$ is the total stress, $\tau$ is the equivalent or effective stress $\tau=\sqrt{(1 / 2) \sigma_{i j}^{\prime} \sigma_{i j}^{\prime}}, \sigma_{i j}^{\prime}=\sigma_{i j}-(1 / 3) \sigma_{k k} \delta_{i j}$, and $\gamma$ is the corresponding equivalent or effective shear strain $\gamma=$ $\sqrt{2 \varepsilon_{i j} \varepsilon_{i j}}$ (with $\varepsilon_{i j}$ denoting here the plastic strain tensor).

\section{Mixture approach}

Along the same lines as for the elastic case, it is assumed that the flow stress $\tau$ of a nanograined polycrystal is made of two parts: the flow stress $\tau_{1}$ of the bulk and the flow stress $\tau_{2}$ of the grain boundary phases, such that

$$
\tau=\tau_{1}+\tau_{2}
$$

In the case of simple shear, the applied stress $\tau^{\text {appl }}$ is carried out by both phases, each of which obeys its own equilibrium equation:

$$
\partial_{x} \tau_{1}=\hat{f} ; \quad \partial_{x} \tau_{2}=-\hat{f} \quad \Rightarrow \quad \partial_{x} \tau=0
$$

where $\hat{f}$ denotes, as before, the exchange of momentum between the two simultaneously deforming "phases" 
under simple shear in a direction normal to the $x$-axis. We further assume that

$$
\tau_{k}=\kappa_{k}\left(\gamma_{k}\right), k=1,2 ; \quad \hat{f}=\alpha\left(\gamma_{1}-\gamma_{2}\right)
$$

where $\kappa_{k}$ and $\gamma_{k}$ denote the flow stress and corresponding shear strain in each one of the two phases, and $\alpha$ is a phenomenological coefficient modeling the exchange of mechanical force between the two phases. If both the strains $\gamma_{k}$ and the gradients $\partial_{x} \gamma_{k}$ are of order $\epsilon$ $(\in \ll 1)$, then the following relation holds:

$$
\begin{aligned}
& {\left[\kappa_{1}^{\prime} \kappa_{2}^{\prime} \partial_{x x}-\alpha\left(\kappa_{1}^{\prime} \partial_{x}+\kappa_{2}^{\prime} \partial_{x}\right)\right] \gamma_{k}=0 \Rightarrow} \\
& \partial_{x x} \delta=\left(\frac{\alpha^{2}\left(\kappa_{1}^{\prime}+\kappa_{2}^{\prime}\right)^{2}}{\kappa_{1}^{\prime 2} \kappa_{2}^{\prime 2}}\right) \delta, \quad \delta \equiv\left(\gamma_{1}-\gamma_{2}\right) / 2
\end{aligned}
$$

where higher order terms in $\in$ have been neglected. On returning to Eq. [15], one can also express it in terms of the total average strain $\gamma=\left(\gamma_{1}+\gamma_{2}\right) / 2$ and $\delta$ as follows:

$$
\tau=\kappa_{1}(\gamma+\delta)+\kappa_{2}(\gamma-\delta)=\kappa(\gamma)-\mu^{*}(\gamma) \delta
$$

where a Taylor expansion was used for the functions $\kappa_{1}$ and $\kappa_{2}$ around $\gamma$ and $\delta=0$, and only terms linear in $\delta$ were retained. By the same argument, it turns out that Eq. $[18]_{2}$ can be written as

$$
\partial_{x x} \gamma=\lambda^{*}(\gamma) \delta
$$

which, upon combination with the above equations, gives

$$
\tau=\kappa(\gamma)-c \partial_{x x} \gamma
$$

where $c$ is, in general, a function of $\gamma$ and relates explicitly to $\lambda^{*}$ and $\mu^{*}$ which, in turn, are given in terms of $\kappa_{1}^{\prime}, \kappa_{2}^{\prime}$ and $\alpha$. We can then write the threedimensional counterpart of Eq. [21] as

$$
\tau=\kappa(\gamma)-c \nabla^{2} \gamma
$$

i.e., the same form at which we have arrived earlier within the bulk/surface approach.

In concluding this section, certain comments on the dependence of the flow stress $\tau$ on the first gradient of the effective strain $\gamma$ would be useful. Such dependence was excluded in Eq. [21] on the basis of a Taylor expansion and isotropy. [The condition $\tau(x)=\tau(-x)$ suggests that the dependence on $\partial_{x} \gamma$ drops and only the dependence on $\partial_{x x} \gamma$ and $\left(\partial_{x} \gamma\right)^{2}$ may be retained up to terms of second degree and order, if the assumption of linearity is relaxed.] An admissible dependence on first gradients is possible by allowing for the absolute value of $\partial_{x} \gamma$, i.e., $|\nabla \gamma|$, to enter in the formulation. For example, the constitutive assumption for $\mathcal{M}$ in Eq. [11] $]_{2}$ may be generalized to read $\mathcal{M}=\partial_{x} \mathrm{~S}-\bar{c}|\nabla \gamma|^{n / 2}$, where $\bar{c}$ and $n$ are constants. The sign of the various gradient coefficients depends on whether the material is under strain hardening (or strain softening) and strain gradient hardening (or strain gradient softening). For strain softening $(d \kappa / d \gamma<0)$, Eq. [22] with $c>0$ was used ${ }^{[2,3]}$ to obtain the thickness of shear bands during localization of deformation. In the absence of localization, the coefficient $\bar{c}$ is taken as positive for strain gradient hardening and negative for strain gradient softening. In this connection, it would be instructive for later purposes to establish contact between the preceding phenomenological arguments and dislocation theory in relation to the role of grain size in controlling material behavior and interpreting size-dependent stress-strain curves. In the absence of localization, during the initial stages of plastic deformation, it easily turns out that the flow stress may be written as $\tau=\tau_{0}+k l^{1 / 2}|\nabla \gamma|^{1 / 2}$ by taking $c=0, \kappa(\gamma)=\tau_{0}$, and $n=1 ; \tau_{0}$ is the homogeneous portion of the flow stress containing frictionlike and other nongradient contributions, $k$ is a stresslike parameter accounting for strain gradient hardening/ softening, and $l$ is an internal length related to dislocation spacing or dislocation source distance. Such square root dependence of $\tau$ on $|\nabla \gamma|^{1 / 2}$ may be physically justified by recalling Taylor's hardening relation $\tau \propto \sqrt{\rho}$, where $\rho$ denotes dislocation density. By splitting the dislocation density to $\rho_{S}$ (density of "statistically distributed" dislocations) and $\rho_{G}$ (density of "geometrically necessary" dislocations), we can write $\tau=A_{S} \sqrt{\rho_{S}}+A_{G} \sqrt{\rho_{G}}=A_{S} \sqrt{\rho_{S}}\left(1+A^{*}|\nabla \gamma|^{1 / 2}\right)$, where the well-known proportionality relationship $\rho_{G} \propto \nabla \gamma$ is used and the $A$ 's are trivially inter-related. By considering slow variations of $\rho_{S}$ as compared to $\rho_{G}$ and properly identifying the various material functions and parameters $\left(\tau_{0}, k, l ; \rho_{S}, A_{S}, A_{G}, A^{*}\right)$, one can easily arrive at the desired result.

\section{Nanodiffusion}

The approach adopted earlier for a deforming $\mathrm{NC}$ or UFG material, viewed as a continuum that can interact with its surface or as a medium consisting of two (bulk and grain boundary) interacting phases, can be applied to model transport processes such as diffusion and heat conduction. The discussion here is focused on (substitutional) diffusion in NC materials for which it has been shown that the diffusivity may be more than ten orders of magnitude higher in comparison to regular bulk lattice diffusion. The two phases that contribute to the overall diffusion process, however, are taken here to correspond to grain boundary or intercrystalline (IC) space (as before) and grain boundary triple junction (TJ) (regular lattice diffusion in the intergranular space is thus neglected). The IC space is characterized by a diffusivity comparable to grain boundary diffusivity of conventional polycrystals and the TJs space is characterized by a diffusivity comparable to conventional surface diffusion. This view may be supported by the fact that the values of activation energies for substitutional diffusion in NC materials are more comparable to surface diffusivities rather than to grain boundary diffusivities in conventional polycrystals. $^{[5,6]}$ For example, at room temperature, the activation energies for diffusion of $\mathrm{Cu}$ and $\mathrm{Ag}$ in $n-\mathrm{Cu}$ are 0.64 and $0.39 \mathrm{eV} /$ atom, while the corresponding activation energies for surface diffusion are 0.69 and $0.30 \mathrm{eV} /$ atom, respectively. 


\section{Bulk/surface approach}

In this approach, the classical Fick's law for diffusion is replaced by

$$
\mathbf{j}=\mathbf{j}^{\mathbf{b}}+\hat{\mathbf{j}} ; \quad \mathbf{j}^{\mathbf{b}}=-D \nabla \rho
$$

where $D$ is the diffusivity of the nc material, $\rho$ is the concentration, $\mathbf{j}^{\mathbf{b}}$ is the usual Fickean flux, and $\hat{\mathbf{j}}$ is an extra flux term to account for the flux exchange between bulk and surface points. In analogy to previous sections for deformation processes, it is assumed that $\hat{\mathbf{j}}$ is determined by a second-order tensor flux or "hyperflux" term $\mathbf{J}$ such that

$$
\hat{\mathbf{j}}=\operatorname{div} \mathbf{J}
$$

In the case of steady states $\left(\partial_{t} \mathbf{j}^{\mathbf{b}}=0\right)$, the hyperflux $\mathbf{J}$ may be assumed to be proportional to the gradient of $\mathbf{j}$ (in analogy to the case of elasticity), while for transient states $\left(\partial_{t} \mathbf{j}^{\mathbf{b}} \neq 0\right)$, the gradient of $\partial_{t} \mathbf{j}^{\mathbf{b}}$ should also be taken into account. Thus, the simplest possible constitutive expression for $\mathbf{J}$ is the following form:

$$
J=\nabla\left(c \mathbf{j}^{\mathbf{b}}+c^{*} \partial_{t} \mathbf{j}^{\mathbf{b}}\right)
$$

which, upon substitution to Eq. [24], gives

$$
\hat{\mathbf{j}}=-D \nabla^{2}\left(c \nabla \rho+c^{*} \partial_{t} \nabla \rho\right)
$$

where the classical Fickean expression of Eq. [23] $]_{2}$ for $\mathbf{j}^{\mathbf{b}}$ was used. Upon substituting Eq. [26] into the standard differential statement expressing conservation of mass,

$$
\partial_{t} \rho+\operatorname{div} \mathbf{j}=0
$$

one obtains

$$
\partial_{t} \rho=D \nabla^{2} \rho+c^{*} \mathrm{D} \partial_{t} \nabla^{2} \rho+c D \nabla^{4} \rho
$$

i.e., a higher-order diffusion equation containing thirdand fourth-order gradients of the concentration $\rho$, in addition to the classical diffusion equation. The new phenomenological coefficients $c$ and $c^{*}$ depend on the nanoscopic configuration (the lifetime of diffusion species in IC and TJ spaces, as well as the spatial characteristics of grain, grain boundary, and triple grain boundary junctions), and they should not be confused with the corresponding gradient coefficients or internal length parameters that have been used earlier in the nanoelasticity and nanoplasticity derivations.

\section{Mixture approach}

A more natural way to reach the preceding conclusion is to assume that Fickean diffusion takes place separately in the "IC" and "TJ" spaces, while the differential statements of mass conservation for each space are modified by a source term to account for the exchange of diffusing species between the two co-existing "media." Such an approach was advanced by the author in the late $1970 \mathrm{~s}^{[7,8]}$ for the transport process in media with double diffusivity and is summarized subsequently for the present case of two distinct diffusivity paths: one through the IC region and another through the TJ channels, which play the role of fast diffusion paths.
By denoting the concentrations in the TJ and IC spaces by $\left(\rho_{1}, \rho_{2}\right)$ and the corresponding fluxes by $\left(\mathbf{j}_{1}, \mathbf{j}_{2}\right)$, the following statements hold:

$$
\begin{array}{r}
\partial_{t} \rho_{1}+\operatorname{div} \mathbf{j}_{1}=\hat{c}_{1} ; \partial_{t} \rho_{2}+\operatorname{div} \mathbf{j}_{2}=\hat{c}_{2} \\
\mathbf{j}_{1}=-D_{1} \nabla \rho_{1} ; \mathbf{j}_{2}=-D_{2} \nabla \rho_{2}
\end{array}
$$

where $\left(\hat{c}_{1}, \hat{c}_{2}\right)$ are source terms representing the local exchange of mass between the TJ and IC spaces. Mass conservation for the total concentration $\rho=\rho_{1}+\rho_{2}$ requires $\hat{c}_{1}=-\hat{c}_{2}=\hat{c}$ (with $\mathbf{j}=\mathbf{j}_{1}+\mathbf{j}_{2}$ representing the total flux). In the simplest possible case, $\hat{c}$ may be expressed as a linear function of the concentrations $\rho_{1}$ and $\rho_{2} ;$ i.e.,

$$
\hat{c}=-\kappa_{1} \rho_{1}+\kappa_{2} \rho_{2}
$$

where $\left(\kappa_{1}, \kappa_{2}\right)$ are phenomenological mass exchange coefficients, which depend on the relevant time and space scales involved and measure the rate at which diffusive atoms in the IC space jump spontaneously to the $\mathrm{TJ}$ space and vice versa.

Combination of Eqs. [29] and [30] yields the following set of coupled diffusion equations:

$$
\begin{aligned}
& \partial_{t} \rho_{1}=D_{1} \nabla^{2} \rho_{1}-\kappa_{1} \rho_{1}+\kappa_{2} \rho_{2} ; \\
& \partial_{t} \rho_{2}=D_{2} \nabla^{2} \rho_{2}-\kappa_{2} \rho_{2}+\kappa_{1} \rho_{1}
\end{aligned}
$$

It then turns out that uncoupling of the preceding equations yields the following higher-order diffusion equation for $\rho$ :

$$
\partial_{t} \rho+\tau \partial_{t}^{2} \rho=D \nabla^{2} \rho+c^{*} D \partial_{t} \nabla^{2} \rho+c D \nabla^{4} \rho
$$

where $\tau=\left(\kappa_{1}+\kappa_{2}\right)^{-1}, \mathrm{D}=\tau\left(\kappa_{1} D_{2}+\kappa_{2} D_{1}\right), c^{*}=\tau\left(D_{1}+\right.$ $\left.D_{2}\right) / D$, and $c \equiv-\tau D_{1} D_{2} / D$. It follows that by neglecting the inertia term $\partial_{t}^{2} \rho$ (slow diffusion processes), Eq. [32] becomes formally identical to Eq. [28]. The extra dividend in this case is the further physical interpretation of the gradient parameters $c$ and $c^{*}$. For large times $(t \rightarrow \infty)$, it turns out that Eq. [28] reduces to the classical diffusion equation; i.e.,

$$
\partial_{t} \rho=D \nabla^{2} \rho ; \quad D \equiv D_{\text {eff }}=\frac{\kappa_{2}}{\kappa_{1}+\kappa_{2}} D_{1}+\frac{\kappa_{1}}{\kappa_{1}+\kappa_{2}} D_{2}
$$

It is worth noting that the effective diffusion coefficient may be expressed as $D \equiv D_{\text {eff }}=f D_{1}+(1-f) D_{2}$, with the identification of the volume fractionlike parameter $f$ as $f=\kappa_{2} /\left(\kappa_{1}+\kappa_{2}\right)$.

\section{BENCHMARK CONFIGURATIONS}

\section{A. Size-Dependent Elastic Moduli}

As an application of the "nanoelasticity" gradientdependent stress-strain relation given by Eq. [9], the dependence of the effective (macroscopic) elastic moduli of NC's on grain size is obtained by solving an elementary boundary value problem.

Let us consider the idealized one-dimensional configuration of Figure 1(a) depicting two adjacent grains of 


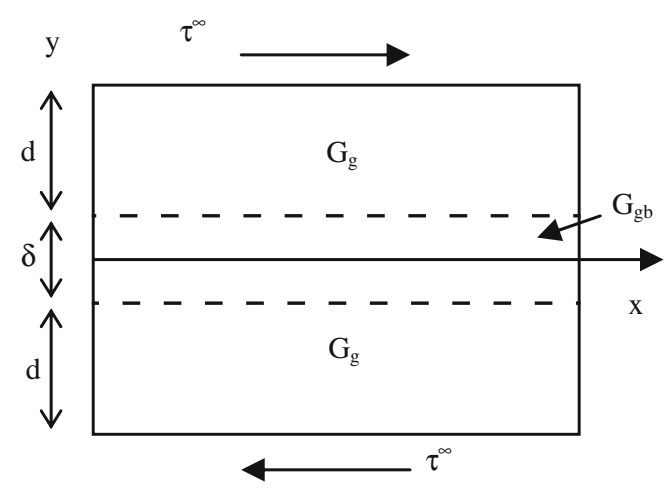

(a)

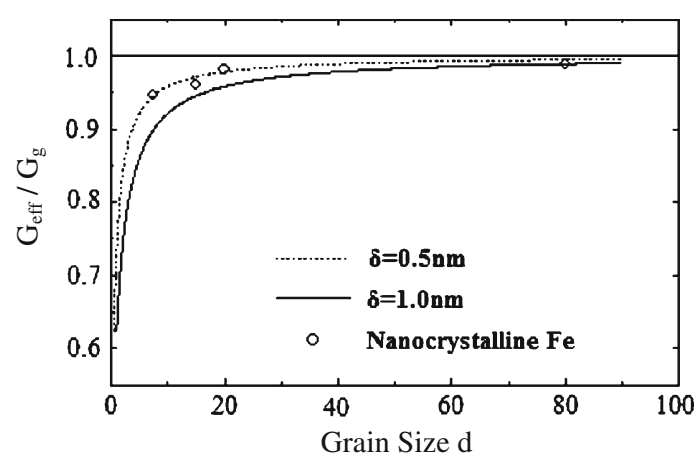

(b)

Fig. 1-(a) Idealized boundary value problem for an elastic NC and $(b)$ solution for the effective shear modulus for two values of grain boundary thickness, as compared with experimental data.

size $d$ connected by a grain boundary of thickness $\delta$ subjected to a shear $\tau^{\infty}$, while different local moduli $G_{g}$ and $G_{g b}$ are assigned to the "grain interior" and the grain boundary regions. The stress-strain relation for both regions is assumed to obey Eq. [9], where the gradient term takes into account possible strain nonuniformities due to the disorder and structural defects (nanopores, ledges) characterizing the nonequilibrium grain boundaries of NC's. Thus, for small elastic deformations, the governing equations of gradient elasticity given by Eq. [5] for the present nanoscale problem are reduced to

$$
\partial_{y} \tau=0 \quad \Rightarrow \quad \tau=\tau^{\infty} ; \quad G \gamma-c \partial^{2} \gamma / \partial y^{2}=\tau^{\infty}
$$

with $(\tau, \gamma)$ denoting shear stress and strain and $G$ being the shear modulus in each one of the three regions shown. The solution of Eq. [34] is straightforward and is left as an exercise to the reader. The auxiliary conditions to be used, in conjunction with the general solution of Eq. [34] $]_{2}$, are

$$
\begin{aligned}
& \left.\partial_{y} \gamma_{g b}\right|_{y=0} ; \gamma_{g}=\gamma_{g b} \text { and } \\
& \partial_{y} \gamma_{g}=\partial_{y} \gamma_{g b} \text { at } y=\delta / 2 ;\left.\gamma_{g}\right|_{y=d+\delta / 2}=\tau^{\infty} / G_{g}
\end{aligned}
$$

where symmetry with respect to $y=0$ is assumed. Then the average shear strain $\bar{\gamma}$ reads

$$
\bar{\gamma}=\frac{1}{(d+\delta / 2)}\left[\int_{0}^{\delta / 2} \gamma_{g b} d y+\int_{\delta / 2}^{d+\delta / 2} \gamma_{g} d y\right] ; \quad G_{e f f} \equiv \tau^{\infty} / \bar{\gamma}
$$

where Eq. $[36]_{2}$ defines an effective shear modulus that may be taken to correspond to the "overall" elastic modulus of the considered nc. The corresponding plot of $G_{\text {eff }} v s d$ is given in Figure 1(b), consistently with the experimental results. ${ }^{[9]}$

It should be emphasized that graphs of the type depicted in Figure 1(b) are obtained by assigning specific values for the gradient coefficients $\left(c_{g}=\right.$ $\left.l_{g}^{2} G_{g}, c_{g b}=l_{g b}^{2} G_{g b}\right)$ and assuming that the internal lengths $l_{g}$ and $l_{g b}$ are comparable in size (of the order 0.25 to $0.75 \mathrm{~nm}$ for $l_{g b}$ and 1 to $2 \mathrm{~nm}$ for $l_{g}$ ), as well as that the shear modulus of the grain boundary space is a fraction of the shear modulus of the grain (e.g., $G_{g b}=$ $0.3-0.75 G_{g}$ ). It may be of interest to point out that the solution for the average strain $\bar{\gamma}$ in Eq. [36] may be written as the sum of a homogeneous part $\left[\tau^{\infty} /\right.$ $(d+\delta / 2)]\left[(\delta / 2) G_{g b}^{-1}+d G_{g}^{-1}\right]$ and an inhomogeneous gradient-dependent term $\tau^{\infty} / G_{\text {grad }}$, where the gradient modulus $G_{\text {grad }}$ depends explicitly on the elastic, internal length, and other geometric parameters of the bicrystal listed previously. It follows then that the effective shear modulus can be written as $G_{\text {eff }}^{-1}=f G_{g}^{-1}+(1-f) G_{g b}^{-1}+$ $G_{\text {grad }}^{-1}$, where $f \equiv f_{g}=d /(d+\delta / 2)$ and $f_{g b}=1-f$ are assumed to approximate reasonably well the volume fractions of the grain and grain boundary space in the present one-dimensional configuration. In other words, the effective shear modulus is expressed as the sum of a classical term (familiar from standard mixture rule arguments) and a gradient term (which corrects mixture rule type relationships and models, in addition, size effects). In fact, it turns out that, for $l_{g}$ of the order of about 10 times larger than $l_{g b}$, the ratio $G_{\text {eff }} / G$ initially decreases, and, after attaining a minimum at a grain size (d) comparable to the internal length $\left(l_{g}\right)$, continues to increase, reaching its asymptotic value (1) for large grain sizes. More details on such type of simple gradient arguments to illustrate the interplay of grain size, grain boundary thickness, and internal length parameters in interpreting stiffening or softening of the shear modulus will be given in a future article, where corresponding size effects for the elastic moduli of nanopolycrystals and other nano-objects (nanolayers, nanowires, and nanotubes) will also be reported.

\section{B. Inverse H-P Effect}

In this section, we discuss normal and abnormal H-P behavior for various material properties as we cross over from the microcrystalline to the nanocrystalline regime. Such H-P effect has traditionally been considered for the yield strength of polycrystals, along with its inverse behavior as the grain size is reduced below a critical value $d_{\text {crit }}$ of the order of a few nanometers. However, recent experimental evidence reveals a similar behavior 
for the activation volume and the pressure-sensitivity parameters. Certain results on this topic are given subsequently (Section III-B-2).

\section{Inverse $H$-P behavior for strength}

The first attempt to provide a quantitative explanation for the inverse HP behavior in NC polycrystals, at the time that such behavior was still under debate (in view of the inadequate then experimental evidence), was provided by the author and co-workers. ${ }^{[10]}$ This was a rather phenomenological model based on the assumption that the strength of $\mathrm{NC}$ polycrystals is given by a simple rule of mixtures relation $\sigma=f \sigma_{g}+(1-f) \sigma_{g b}$, which, in view of Tabor's empirical relation for the hardness $\left(H \sim 3 \sigma_{y s}\right)$, may be written as

$$
H=f H_{g}+(1-f) H_{g b}
$$

where $f$ denotes volume fraction, $H_{g}=H_{g}^{0}+k_{g} / \sqrt{d}$ is given by the classical $\mathrm{H}-\mathrm{P}$ relation, and $H_{g b}$ is the hardness of the grain boundary taken equal to that of the corresponding amorphous state. A more elaborate model based on Eq. [37], but assuming that both $H_{g}$ and $H_{g b}$ are given by the standard $\mathrm{H}-\mathrm{P}$ relation, was discussed in Reference 11 by also using a dislocation mechanism-based argument (Orowan bypassing) to relate $k_{g}$ and $k_{g b}$ of the grain and the grain boundary strength constants. [The volume fraction $f$ was approximated there by a three-dimensional expression of the form $f=(d-\delta)^{3} / d^{3}$, with $d$ denoting the grain size and $\delta$ the grain boundary width, which was taken to be of the order of $1 \mathrm{~nm}$.] The final expression for the hardness $H$ as a function of the grain size $d$ was

$$
H=H_{o g}+k_{g} F(d) d^{-1 / 2}
$$

where $F(d)=(1-\delta / d)^{3}+\left[1-(1-\delta / d)^{3}\right]\left[\ln \left(\theta d / r_{0}\right) / \ln \left(\theta d_{c} / r_{0}\right)\right]$, with $\theta(<1)$ being an adjustable parameter and $d_{c}=d_{\text {crit }}$ denoting the critical grain size where the slope of the $H$ $v s d$ changes sign $\left(r_{0}\right.$ is the distance used in classical dislocation theory to approximate the extent of dislocation core).

The ability of Eq. [38] to fit experimental data is shown in Figure 2 for a number of metals and additional results can be found in the original reference, ${ }^{[11]}$ along with the values of the material parameters used.

Before we proceed with an illustration of how gradient effects could explicitly be incorporated in Eq. [38], we shall point out that the starting averaging relation, as expressed by the standard mixture rule statement given by Eq. [37], may also be interpreted on the basis of a simple gradient argument for the hardness. To this end, we consider in one dimension a grain of size $d$ constrained on its left and right side by two boundaries, each of thickness $\delta$. The corresponding unit cell is then of size $d+\delta$, containing the grain and the two halves of its left and right boundaries. The hardness $H$ of the unit cell is assumed to be the sum of the local hardness $H_{G}$ at the center of the grain plus the gradient contribution due to the heterogeneous distribution of hardness across the unit cell, i.e., $H=H_{G}+l \nabla H_{G}$, where $l$ is an internal length. The gradient of the local hardness $\nabla H_{G}$ can be written, in a first approximation, as twice (in order to account for the left and right contribution $)$ the quantity $\left(H_{G B}-H_{G}\right) /[(d+\delta) / 2]$, where $H_{G B}$ denotes the hardness at the center of the grain boundary and $(d+\delta) / 2$ is the distance between the centers of the grain and its grain boundary. By assuming then that $l=\delta / 4$, the final expression for the total hardness $H$ becomes $H=\left(d H_{G}+\delta H_{G B}\right) /(d+\delta)$, which is precisely Eq. [37].

In connection with the preceding discussion, it should be noted that a large number of articles have been devoted to rationalizing the standard and the inverse $\mathrm{H}-\mathrm{P}$ relation on the basis of various hypotheses and mechanisms ${ }^{[12-14]}$ and references quoted therein. A rather interesting approach on the basis of gradient theory with surface/interface energy was provided recently in References 15 and 16. The governing

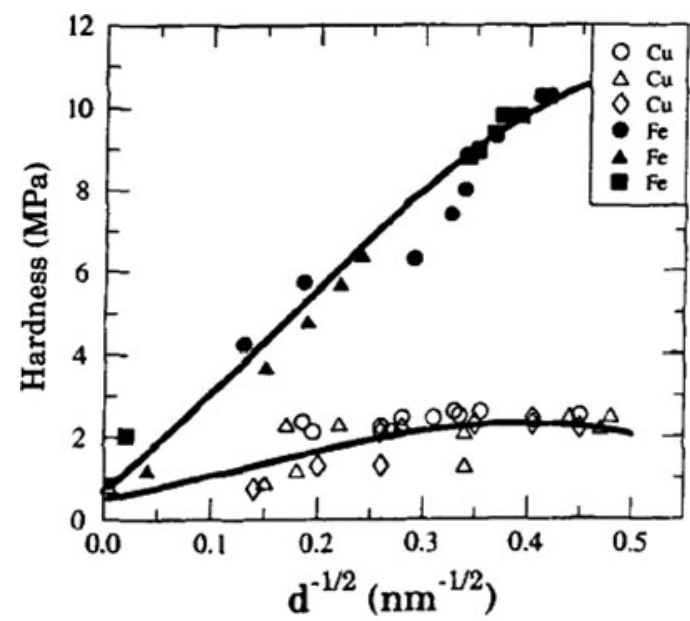

(a)

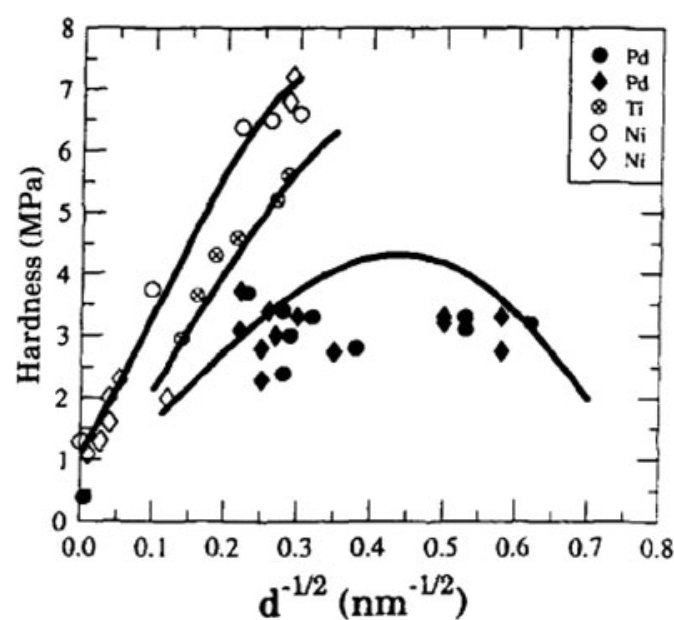

(b)

Fig. 2-Hardness dependence on grain size for NC metals. Additional curves fitting experimental data for a variety of NC materials can be found in the original reference. ${ }^{[1]}$ 


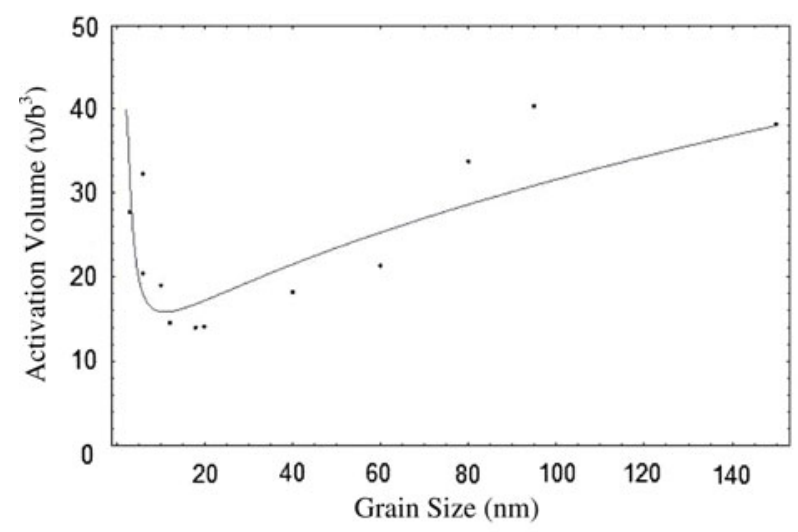

(a)

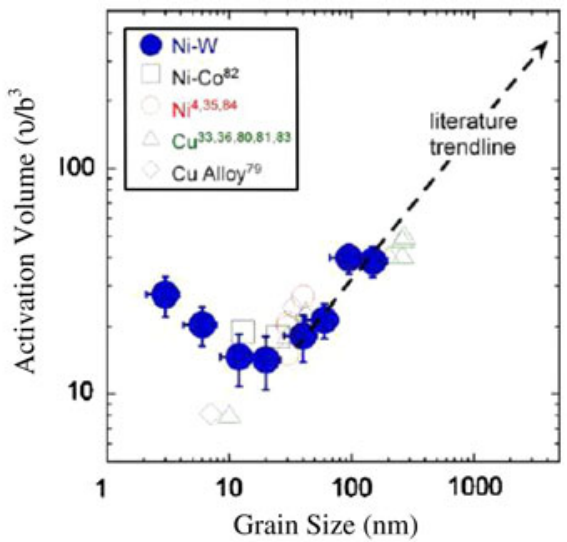

(b)

Fig. 3-Activation volume dependence on grain size for NC materials. The solid line in $(a)$ is a fit of the rule of mixtures relationship, whereas the dots correspond to the experimental graph ${ }^{[17]}$ depicted in $(b)$.

relationship for the strength of the $\mathrm{NC}$ polycrystal in this model is obtained as $H=H_{\mathrm{o}}+k d^{-1 / 2}-$ $\left(\gamma_{g b} / 2 a\right) d^{-1}$, where the first two terms designate a normal H-P dependence and the last term designates an abnormal one $\left(\gamma_{g b}\right.$ and $a$ are material parameters related to grain boundary interfacial energy and geometry). It then turns out easily that the critical grain size that the normal H-P relation breaks down is given by $d_{\text {crit }}=\left(\gamma_{g b} / 2 a\right)^{2}$, and a number of experimental graphs for normal and abnormal H-P behavior can be fitted. In concluding, it is pointed out that such a $d^{-1}$ dependence of strength was arrived at by other authors ${ }^{[12,13]}$ through different arguments.

\section{Inverse H-P Behavior for the Activation Volume} and Pressure Sensitivity

In this section, we provide some preliminary evidence of the possibility of extending the previous (based on an implicit incorporation of gradients) averaging argument for a unit cell (containing a representative grain surrounded by its two halves grain boundaries) to other constitutive parameters at the nanoscale. An explicit incorporation of gradients and surface effects, along the lines of the discussion given in the last paragraph of the preceding section, will be elaborated upon in a future publication. For the purposes of the present article, it suffices to show that the rule of mixtures relation given by Eq. [37] has interesting implications to the physical parameters of activation volume (v) and pressure sensitivity $(\alpha)$, which have recently been considered by several authors for the case of nanophase and amorphous solids (for example, the recent overview by Schuh et al., ${ }^{[17]}$ as well as earlier analogous considerations by the author and co-workers $\left.{ }^{[18,19]}\right)$. The activation volume $v$ is defined by the well-known relation

$$
v=\sqrt{3} k T \frac{\partial \ln (\dot{\varepsilon})}{\partial \sigma}
$$

where $k$ is Bolztmann's constant, $T$ denotes temperature, $\dot{\varepsilon}$ is the strain rate, and $\sigma$ denotes the stress. On denoting the activation volumes for the grain and the grain boundary space by $v_{g}$ and $v_{g b}$, and assuming the simple mixture rule,

$$
\frac{1}{v}=f \frac{1}{v_{g}}+(1-f) \frac{1}{v_{g b}}
$$

with $f=(d-\delta)^{3} / d^{3}$ and $\left(1 / v_{g}\right)=\left(1 / v_{g}^{0}\right)+k_{g} d^{-1 / 2}$, one can deduce the graph of Figure 3(a), which interestingly enough seems to capture the experimental trends depicted in Figure 3(b).

The parameter values used for the model prediction (solid line) and its comparison with the experimental data (dots) are $v_{g}^{0}=1000 \mathbf{b}^{3}, v_{g b}=30 \mathbf{b}^{3}, \delta=2 \mathrm{~nm}$, and $k_{g}=0.3 \sqrt{n m} / \hat{\mathbf{b}}^{3} ; \mathbf{b}$ denotes, as usual, the magnitude of the Burgers vector.

A similar argument may be applied to the pressuresensitive parameter $(\alpha)$ entering the Mohr-Coulomb criterion or, equivalently, the Drucker-Prager yield condition as employed, for example, earlier by the author and his co-workers for UFG materials. ${ }^{[18,19]} \mathrm{On}$ assuming a simple mixture relation of the form $\alpha=f \alpha_{g}+(1-f) \alpha_{g b}$, with $f=(d-\delta)^{3} / d^{3}$ as before, $\alpha_{g b}=$ const., and $\alpha_{g}$ obeying a H-P type relation of the form $\alpha_{g}=\alpha_{g}^{0}+k_{g} d^{-1 / 2}$, one obtains

$$
\alpha=\left(\frac{d-\delta}{d}\right)^{3}\left(\alpha_{g}^{0}+k_{g} d^{-\frac{1}{2}}\right)+\left[1-\left(\frac{d-\delta}{d}\right)^{3}\right] \alpha_{g b}
$$

which, with $\alpha_{g}^{0}=0.02, \alpha_{g b}=0.16, \delta=2 \mathrm{~nm}$, and $k_{g}=07 \sqrt{\mathrm{nm}}$, leads to the model prediction given by the solid line of Figure 4(a) and its comparison with the experiments (dots), as detailed in Figure 4(b) and related simulations. $^{[17]}$

\section{Curvature in Concentration-Penetration Depth Diffusion Profiles}

As an application of the "nanodiffusion" higher-order gradient-dependent transport relation given by Eq. [31] or [32], the concentration depth profile for NC's is obtained by solving an elementary boundary value problem. In fact, solutions to Eqs. [31] and [32] were 


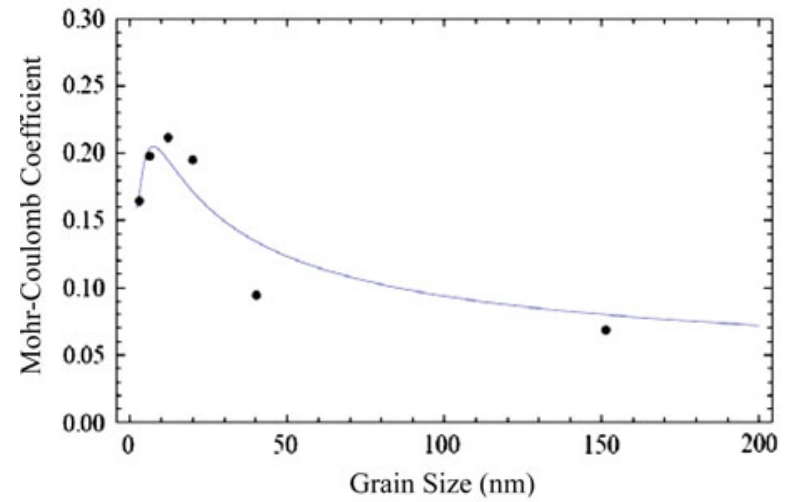

(a)

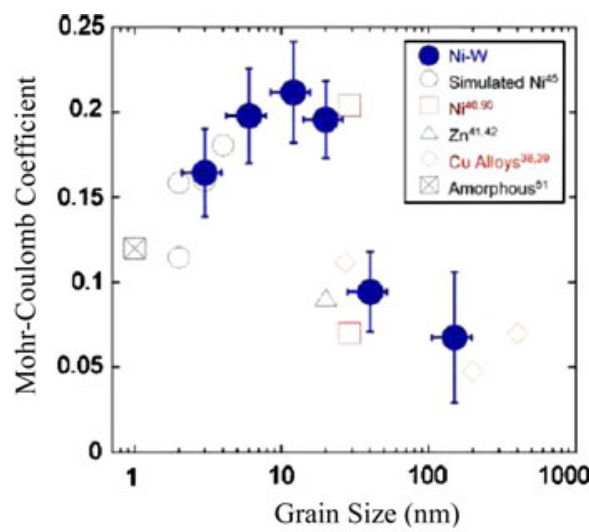

(b)

Fig. 4-Pressure-sensitivity parameter dependence on grain size for NC materials. The solid line in $(a)$ is a fit of the rule of mixtures relationship, whereas the dots correspond to the experimental $\operatorname{graph}^{[17]}$ depicted in $(b)$.

obtained earlier in terms of solutions of the classical diffusion equation. ${ }^{[20,21]}$ For example, for a one-dimensional semi-infinite configuration, the concentration $\rho_{1}$ along the short circuit $\mathrm{TJ}$ channels and the concentration $\rho_{2}$ along the IC region turn out to be given by the formulas

$$
\begin{aligned}
\rho_{1}= & e^{-\kappa_{1} t} h_{1}\left(x, D_{1} t\right)+\frac{\sqrt{\kappa_{2}}}{\left(D_{1}-D_{2}\right)} e^{\lambda t} \\
& \times \int_{D_{2} t}^{D_{1} t} e^{-\mu \xi}\left[A_{11} h_{1}(x, \xi)+A_{12} h_{2}(x, \xi)\right] d \xi \\
\rho_{2}= & e^{-\kappa_{2} t} h_{2}\left(x, D_{2} t\right)+\frac{\sqrt{\kappa_{1}}}{\left(D_{1}-D_{2}\right)} e^{\lambda t} \\
& \times \int_{D_{2} t}^{D_{1} t} e^{-\mu \xi}\left[A_{22} h_{2}(x, \xi)+A_{21} h_{1}(x, \xi)\right] d \xi \\
A_{11}= & \sqrt{\kappa_{1}}\left(\frac{\xi-D_{2} t}{D_{1} t-\xi}\right)^{\frac{1}{2}} \mathrm{I}_{1}(\eta), A_{12}=\sqrt{\kappa_{2}} \mathrm{I}_{0}(\eta), \\
A_{22}= & \sqrt{\kappa_{2}}\left(\frac{D_{1} t-\xi}{\xi-D_{2} t}\right)^{\frac{1}{2}} \mathrm{I}_{1}(\eta), A_{21}=\sqrt{\kappa_{1}} \mathrm{I}_{0}(\eta) \\
\lambda= & \frac{\kappa_{1} D_{2}-\kappa_{2} D_{1}}{D_{1}-D_{2}}, \quad \mu=\frac{\kappa_{1}-\kappa_{2}}{D_{1}-D_{2}}, \\
\eta= & \frac{2 \sqrt{\kappa_{1} \kappa_{2}}}{D_{1}-D_{2}}\left[\left(D_{1} t-\xi\right)\left(\xi-D_{2} t\right)\right]^{\frac{1}{2}}
\end{aligned}
$$

where $\left(I_{0}, I_{1}\right)$ denote modified Bessel functions, and the functions $h_{1}$ and $h_{2}$ obey the classical diffusion equation, i.e., $\partial_{t} h_{k}=\nabla^{2} h_{k} ; k=1,2$. In other words, for a finite source (thin film) initial condition, we have $h_{k}=\left(m_{k} / 2 \sqrt{\pi t}\right) \exp \left(-x^{2} / 4 t\right)$, while for a constant source (thick film) initial condition, we have $h_{k}=c_{k}^{0} \operatorname{erfc}(x / 2 \sqrt{t}) ; m_{k}$ denotes the initial amount of mass and $c_{k}^{0}$ the initial concentrations at each diffusion path. It then turns out that the analytically derived concentration-penetration depth $\left(\log c-x^{2}\right)$ profiles can model the curvature exhibited by the experimental graphs obtained from diffusion data in NC polycrystals, as shown in Figure 5, by properly adjusting the model parameters ( $D$ 's and $\kappa$ 's). Such profiles cannot be obtained by using Fickean diffusion arguments, which lead only to linear plots. More details along these lines can be found in an earlier work of the double diffusivity model applied to substitutional transport in polycrystals. ${ }^{[22]}$

\section{REVISITING DISLOCATION AND FRACTURE THEORY}

In this section, we discuss the possibility of revisiting classical dislocation and fracture mechanics theory based on linear elasticity by considering certain typical problems within the extended gradient elasticity framework summarized by Eqs. [5]. This is done because at the nanoscale an additional size/internal length dependence (other than the Burgers vector magnitude " $b$ " for dislocation problems, and the crack length " $a$ " for fracture problems) may determine mechanical behavior. Elasticity solutions for dislocation and crack problems were used with great success for interpreting mechanical behavior at meso- and micro-scales for a plethora of geometric and loading configurations, as well as a variety of deformation mechanisms. Since gradient elasticity brings in an additional internal length in a general way, independently of the details of the underlying submicroscopic configurations and related deformation mechanisms, it is suggested that the derivation and use of new modified gradient elasticity formulas may provide a new tool for interpreting behavior at the nanoscale.

\section{A. Stability of an Intragrain Dislocation}

As has been shown elsewhere, ${ }^{[23-26]}$ the self-energy per unit length of a screw dislocation within the gradient theory of nanoelasticity summarized by Eqs. [5] is given by the expression

$$
w=\frac{G b^{2}}{4 \pi}\left[\ln \frac{R}{2 \sqrt{c}}+\gamma^{E}+2 K_{0}\left(\frac{R}{\sqrt{c}}\right)\right]
$$

where $R$ is the radial coordinate defining the material volume surrounding the dislocation line under consideration, $\gamma^{E}$ is the Euler constant, $K_{0}$ denotes the Bessel function, and the rest of the symbols have their usual 

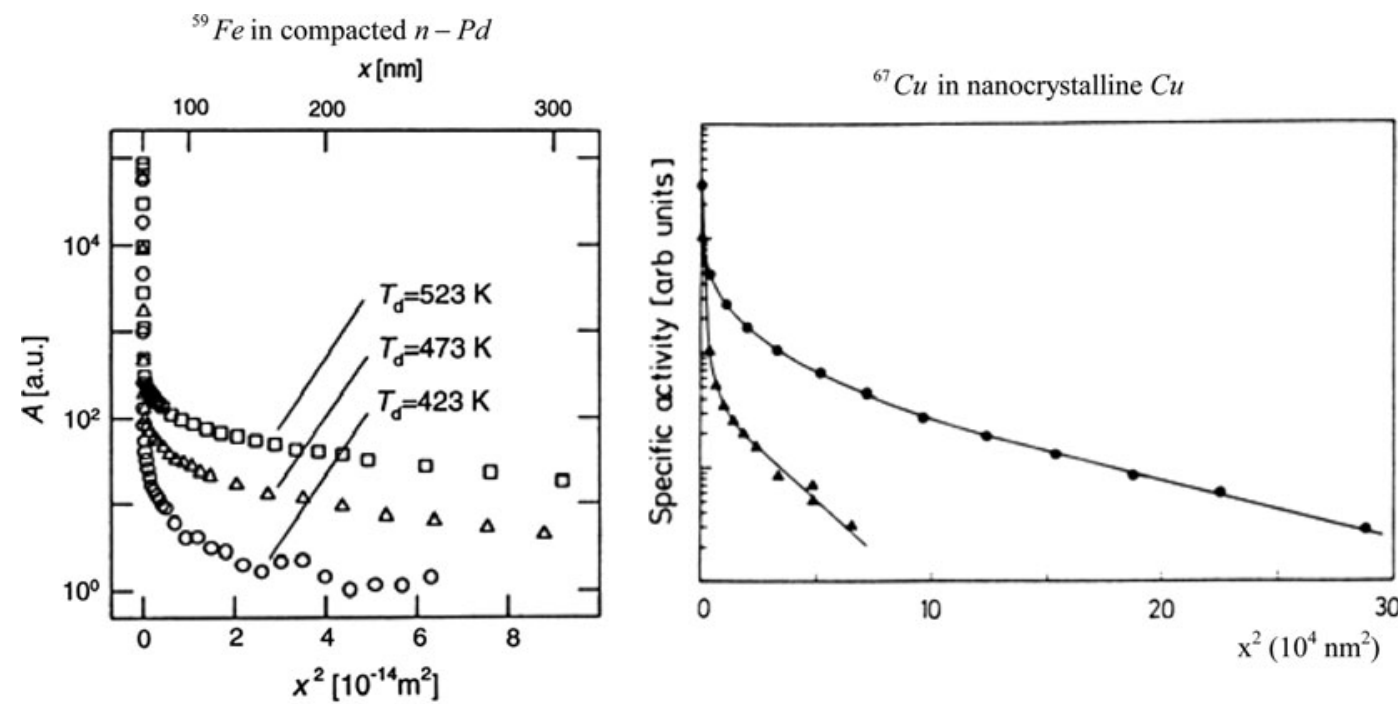

${ }^{18} \mathrm{O}$ in nano $\mathrm{ZrO}_{2}$

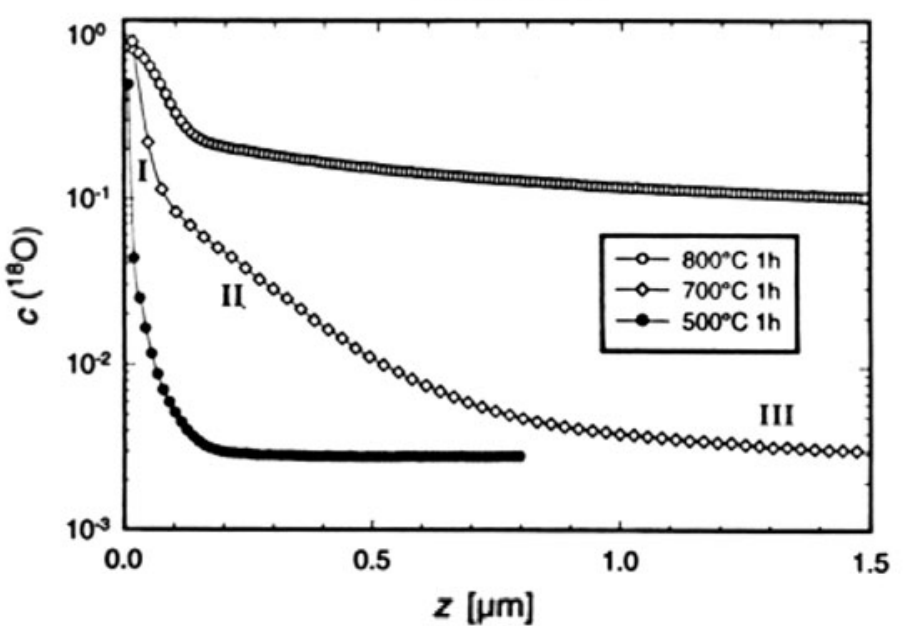

Fig. 5-Experimental concentration-depth penetration profiles in various NC diffusion systems. It turns out that the expressions given by Eq. [42] can fit these experimental profiles by properly adjusting the diffusivities $\left(D_{1}, D_{2}\right)$ and the mass exchange coefficients $\left(\kappa_{1}, \kappa_{2}\right)$.

meaning. It is noted that the self-energy is not singular as in classical elasticity and the necessity of introducing an arbitrary dislocation core parameter for dispensing with such singularity is eliminated. In fact, the gradient coefficient $c$ (its square root denotes the relevant material internal length) provides a new possibility to account for dislocation core effects, as discussed in Reference 24 and the related bibliography listed there. On using this gradient modification of the self-energy in relation to a standard image force argument, ${ }^{[25,26]}$ we obtain the following gradient-dependent expression for the image force $\tau \mathrm{b}$

$$
\tau \mathrm{b}=\frac{G \mathrm{~b}^{2}}{2 \pi}\left[\frac{1}{d}-\frac{1}{2 \sqrt{c}} K_{1}\left(\frac{d}{2 \sqrt{c}}\right)\right]
$$

for a dislocation sitting at the center of a grain of diameter $d$, where $K_{1}$ again denotes the appropriate Bessel function. The plot of the dimensionless stress quantity $\tau / G$ over the dimensionless grain size quantity $d / \sqrt{c}$ is given in Figure 6, when the gradient coefficient (c) or the internal length $(l=\sqrt{c})$ is properly adjusted or scaled with the grain size $(d)$ for the plotted graph to exhibit a maximum, as shown in the figure. This qualitative, and approximately derived under special conditions, plot may be used for the interpretation of the "standard" and "inverse" H-P behavior, as will be discussed more rigorously and in detail, elsewhere. Here, it only suffices to note that for $l \rightarrow 0$, the classical expression for the image force is recovered, and for $d \rightarrow \infty$, the image force becomes vanishingly small.

\section{B. Elimination of Singularities at the Tips of Nanocracks}

In the second part of this section, it is shown how gradient elasticity can be used to eliminate the crack tip stress singularity at the nanoscale. It turns out ${ }^{[23-26]}$ that for a loaded NC or UFG material weakened by a (nano) crack, the stress tensor $\sigma_{i j}$ is given by the inhomogeneous Helmholtz equation:

$$
\sigma_{i j}-c \nabla^{2} \sigma_{i j}=\sigma_{i j}^{0}
$$

where $\sigma_{i j}^{0}$ is the classical elasticity solution with the well-known $r^{-1 / 2}$ singularity. For example, the $\sigma_{22}$ component is determined from the differentiated equation: 


$$
\sigma_{22}-c \nabla^{2} \sigma_{22}=\sigma_{22}^{0}=\frac{K_{I}}{\sqrt{2 \pi r}}\left[\cos \frac{\theta}{2}\left(1+\sin \frac{\theta}{2} \sin \frac{3 \theta}{2}\right)\right]
$$

where $K_{I}=\sigma^{\infty} \sqrt{\pi a}$ is the usual stress intensity factor for mode I ( $\sigma^{\infty}$ is the applied tensile stress, and $a$ is the half crack length) and $(r, \theta)$ are the usual polar coordinates with origin at the crack tip. To proceed further, we write the angular component in Eq. [46] as $[(5 / 4) \cos \theta / 2-(1 / 4) \cos 5 \theta / 2]$ and split the obtained equation in two parts. Next, we solve the two resulting inhomogeneous Helmholtz equations separately, employ superposition, and finally take into account the boundary conditions $\sigma_{i j} \rightarrow \sigma_{i j}^{0}$ as $r \rightarrow \infty$ and $r \rightarrow 0$. It then turns out that the desired nonsingular solution can be cast in the form

$$
\sigma_{22}=\frac{K_{I}}{\sqrt{2 \pi r}}\left[\cos \frac{\theta}{2}\left(1+\sin \frac{\theta}{2} \sin \frac{3 \theta}{2}\right)\right]\left(1-e^{-r / \sqrt{c}}\right)
$$

the distribution of which is given in Figure 7, where scaled quantities $a \rightarrow a / \sqrt{c}$ and $r \rightarrow r / \sqrt{c}$ were used in the plots. Nonasymptotic results and more complete expressions for all components of stress and strain, including comparisons with related crack-tip solutions obtained recently by other authors based on

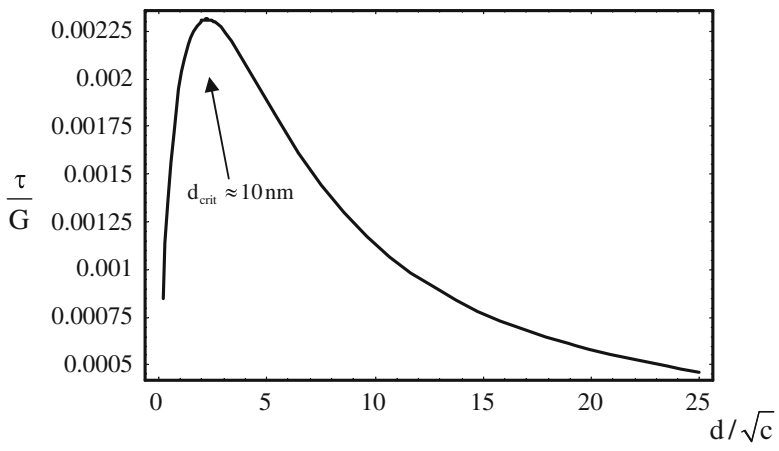

Fig. 6-Plot of the dimensionless stress quantity $\tau / G$ over the dimensionless grain size quantity $d / \sqrt{c}$. The critical grain size where a maximum of $\tau / G$ occurs is $d_{\text {crit }} \approx 10 \mathrm{~nm}$, for the special conditions imposed here in order a maximum to exist. generalizations of the classical elasticity model, will be given elsewhere.

\section{SIZE-DEPENDENT STRESS-STRAIN CURVES}

A mixture rule argument was used recently ${ }^{[15,16]}$ to model the yield stress and full stress-stain graphs for nc metals with varying grain size. This modeling effort was based on a Voce's type constitutive relation for the flow stress, along with a H-P type dependence on the grain size, within a gradient plasticity framework with interfacial energy. ${ }^{[27,28]}$ An alternative procedure is to adopt both an H-P and an inverse H-P dependence for the three stresslike constitutive quantities $\left(\sigma_{s}, \sigma_{f}, h\right)$ appearing in the following Voce-type stress-strain relationship:

$$
\sigma=\sigma_{f}+\left(\sigma_{s}-\sigma_{f}\right) \tanh \left(\frac{h \varepsilon^{p}}{\sigma_{s}-\sigma_{f}}\right)
$$

In particular, we assume the following grain-size dependence for these quantities:

$$
\sigma_{s, f}=\sigma_{s, f}^{o}+k_{s, f} d^{-1 / 2} \text { and } h=h_{0}-k_{h} d^{-1 / 2}
$$

i.e., a normal H-P behavior for $\sigma_{s, f}$ and an inverse one for $h$. In terms of the gradient dependence for the flow stress discussed at the end of Section II-B for strain and strain gradient hardening, i.e., $\sigma=\sigma_{0}+k_{\sigma}^{*} l^{1 / 2}|\nabla \varepsilon|^{1 / 2}$, an $\mathrm{H}-\mathrm{P}$ dependence may be justified by the following dimensionless argument. At the scale of the grain, $|\nabla \varepsilon|$ may be approximated by $\left(\varepsilon_{0} / d\right)^{1 / 2}$, where $\varepsilon_{0}$ is a reference strain and $d$ the grain size. By redefining $k_{\sigma}^{*}$ as $k_{\sigma}^{*} l^{1 / 2} \varepsilon_{0}^{1 / 2}$, the aforementioned size-dependent expressions for $\sigma_{s}$ and $\sigma_{f}$ can be concluded. [An inverse H-P relation for $\sigma_{s}$ may be deduced when the grain boundary phase is softer than the bulk.] For the hardening-like modulus $h$, similar relationships may be concluded by assuming that the grain boundaries act as obstacles to dislocation motion in the bulk (grain boundary hardening), or that the bulk acts as an obstacle to the motion of grain boundary dislocations, a process which is dominant at the nanoscale. In that case (grain boundary softening), the hardening-like modulus $h$ has the form $h=$ $h_{0}-k_{h}^{*} l_{h}^{1 / 2}|\nabla \varepsilon|^{1 / 2}$. Then, Eq. [49] may be concluded,

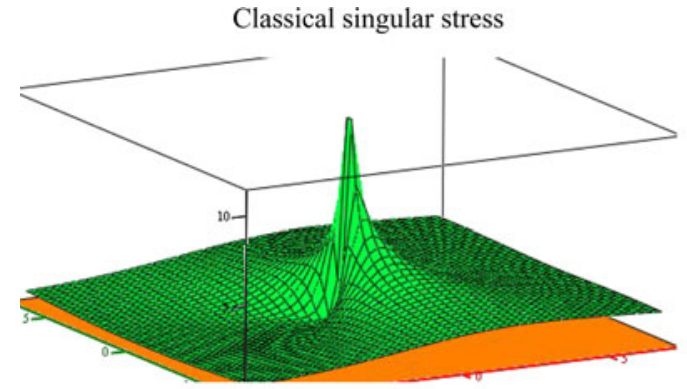

(a)
Gradient non-singular stress

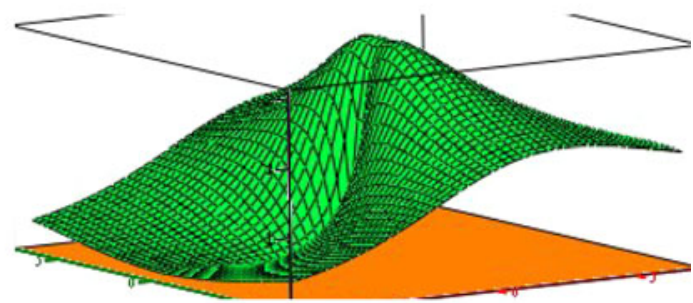

(b)

Fig. 7-Distribution of $\sigma_{22}$ stress in the crack tip region: (a) classical singular stress according to Eq. [46] and (b) gradient nonsingular stress according to Eq. [47]. 
with $k_{s, f}$ and $k_{h}$ directly related to those earlier introduced in this paragraph to facilitate the discussion.

With the preceding assumptions for $\left(\sigma_{s}, \sigma_{f}, h\right)$, one obtains the model predictions depicted in Figure 8(a) (solid lines) and their comparison with experimental data, ${ }^{[29]}$ as detailed in Figure $8(\mathrm{~b})$. The parameter values used are $\sigma_{f}^{0}=70 \mathrm{MPa}, k_{f}=104 \mathrm{kPa} \sqrt{\mathrm{m}}, \sigma_{s}^{0}=$ $230 \mathrm{MPa}, k_{s}=92.5 \mathrm{kPa} \sqrt{\mathrm{m}}, h_{0}=827 \mathrm{MPa}$, and $k_{h}=$ $86 \mathrm{kPa} \sqrt{\mathrm{m}}$.

The same expression for the flow stress, i.e., Eq. [48], can be used for modeling simulation results for smaller grain sizes, where an inverse H-P behavior was documented, ${ }^{[30]}$ as shown in Figure 9.

The parameter values used for the fits of Figure 9(a) are $\sigma_{f}=0.5 \mathrm{GPa}, \sigma_{s}^{0}=4 \mathrm{GPa}, k_{s}=-140 \mathrm{kPa} \sqrt{\mathrm{m}}, h_{0}=$ $730 \mathrm{GPa}$, and $k_{h}=34 \mathrm{MPa} \sqrt{\mathrm{m}}$. The fits in Figures 8 and 9 are discussed in detail in a ERC/LMM-AUT report, ${ }^{[31]}$ where various other possibilities on grain size dependence and gradient-type constitutive equations for nanopolycrystals are examined. In particular, the temperature and strain rate dependence of stress-strain relationships discussed subsequently is also elaborated upon in detail in the aforementioned report, and pertinent results, in comparison with related experiments and simulations, will be published elsewhere.
For the purposes of the present article, however, it suffices to focus on the relevance of the Voce's type constitutive relation given by Eq. [48] for NC polycrystals in interpreting strain rate and temperature variation tests. This is shown by extending Eq. [48] to include a multiplicative strain rate-dependent term of the form $\left\{1+m \ln \left(\dot{\varepsilon} / \dot{\varepsilon}_{0}\right)\right\}$, as well as a multiplicative temperature-dependent term of the form $\left\{1-\left[\left(T-T_{r}\right) /\right.\right.$ $\left.\left.\left(T_{m}-T_{r}\right)\right]^{q}\right\}$ in the overall expression for the flow stress $\sigma$. Related fits to available experimental data are shown in Figures 10 and 11.

Figure 10 shows $\sigma-\varepsilon$ fits under varying $\dot{\varepsilon}$ and $T$ for bulk nc-Cu with grain size of $32 \mathrm{~nm} \cdot{ }^{[29]}$ The expression for $\sigma_{s}$ used for fitting the results of Figure 10(a) is $\sigma_{s}=[890+15 \ln \dot{\varepsilon}] \mathrm{MPa}$, while $\sigma_{f}=383 \mathrm{MPa}$ and $h=$ $96 \mathrm{GPa}$; also, $\dot{\varepsilon}_{0}=1 \mathrm{~s}^{-1}$ and $m=0.017$. The parameter values used for fitting the results of Figure 10(b) are $T_{r}=296 \mathrm{~K}$ and $T_{m}=1356 \mathrm{~K}$ and $q=2.6$. The fits are shown in Figures 10(a)* and (b)*, where solid lines correspond to model predictions and dots correspond to experimental data.

The ability of a Voce's type constitutive relation given by Eq. [48] to model simultaneously the grain size and strain rate dependence is shown in Figure 11. The appropriate grain size dependence of the constitutive

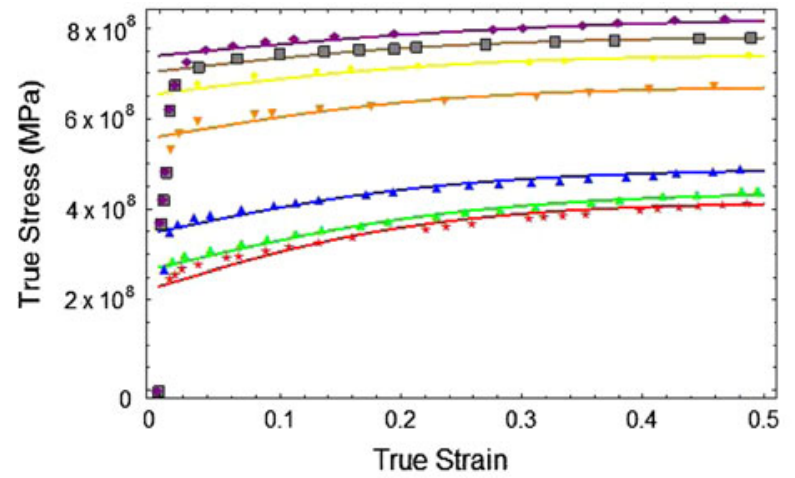

(a)

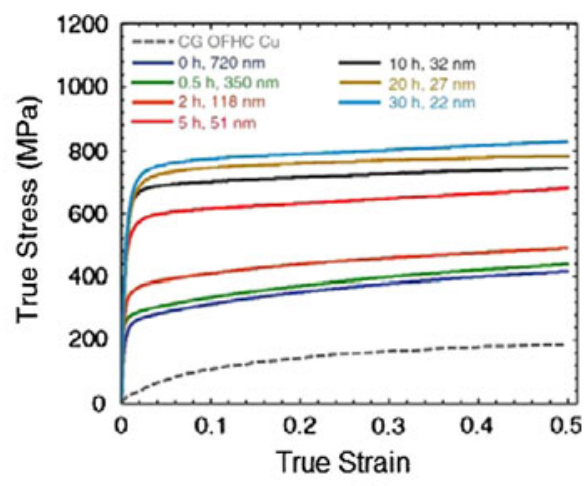

(b)

Fig. 8 - Comparison between model predictions and experimental data for nc-Cu samples of varying grain size.

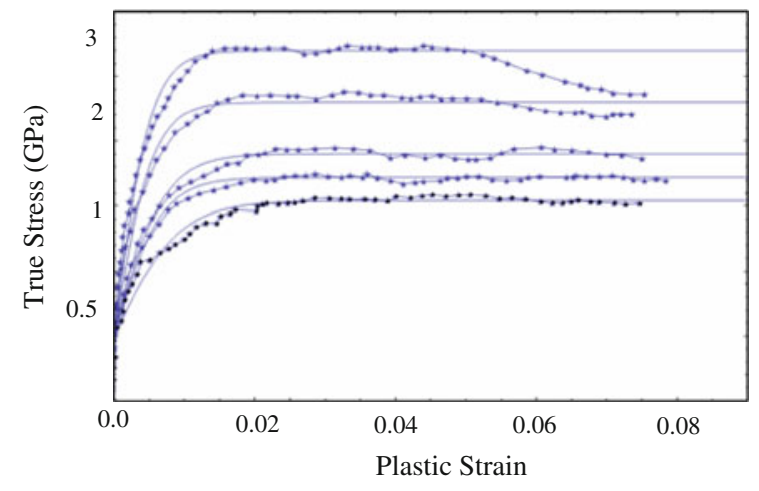

(a)

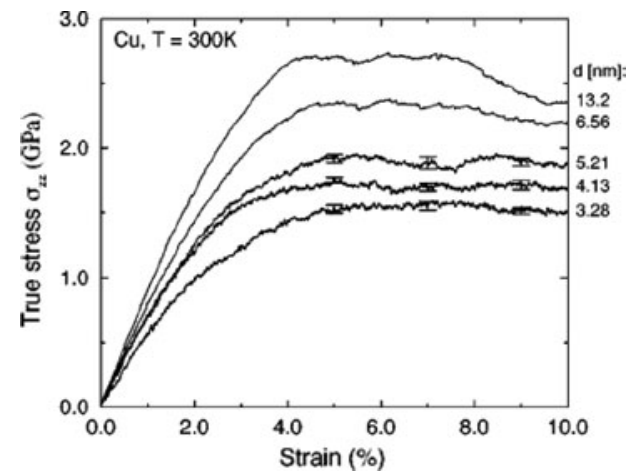

(b)

Fig. 9-Comparison between model predictions and simulations for nc- $\mathrm{Cu}$ samples of varying grain size. 


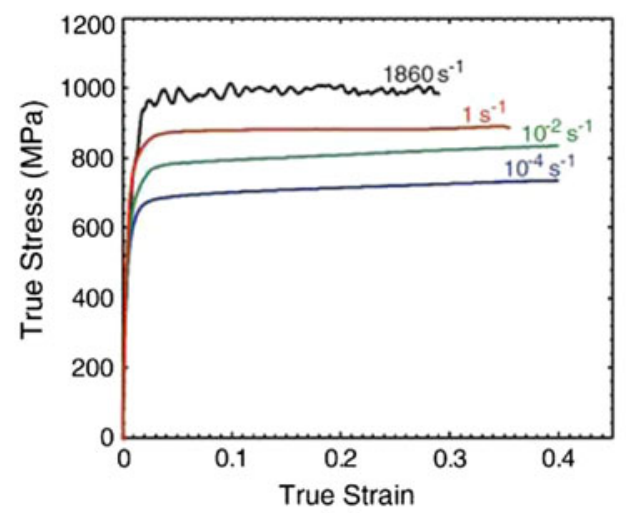

(a)

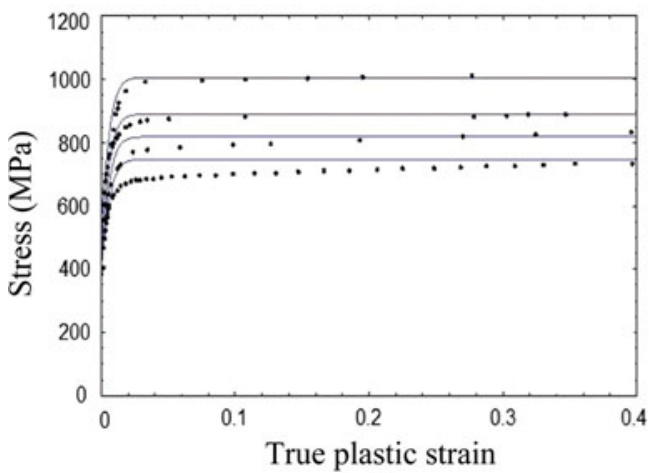

$\left(a^{*}\right)$

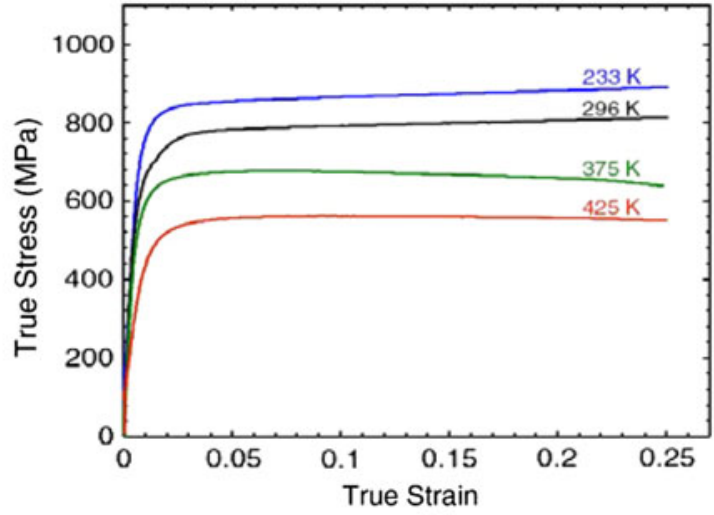

(b)

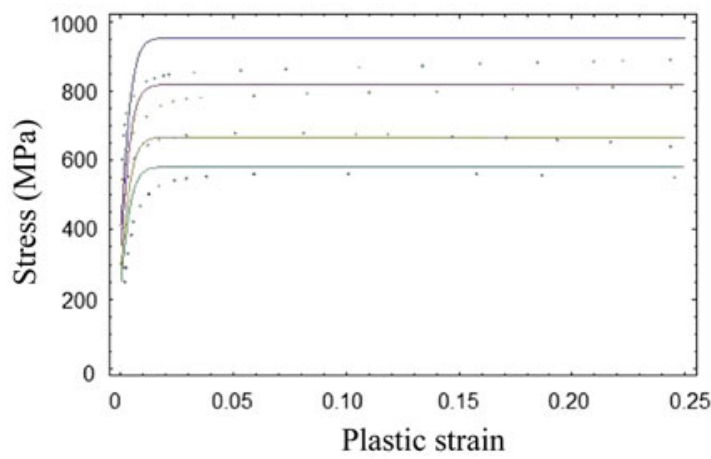

(b*)

Fig. 10 - Strain rate and temperature effects on the $\sigma-\varepsilon$ relation for nc-Cu: $(a)$ and $(b)$ Experimental graphs; $\left(a^{*}\right)$ and $\left(b^{*}\right)$ Model predictions.

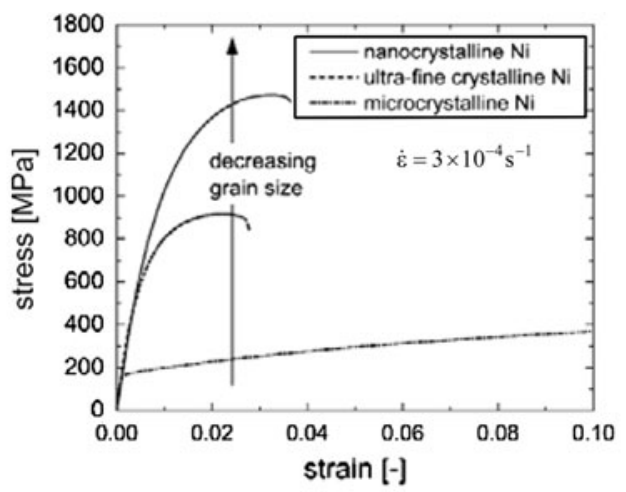

(a)

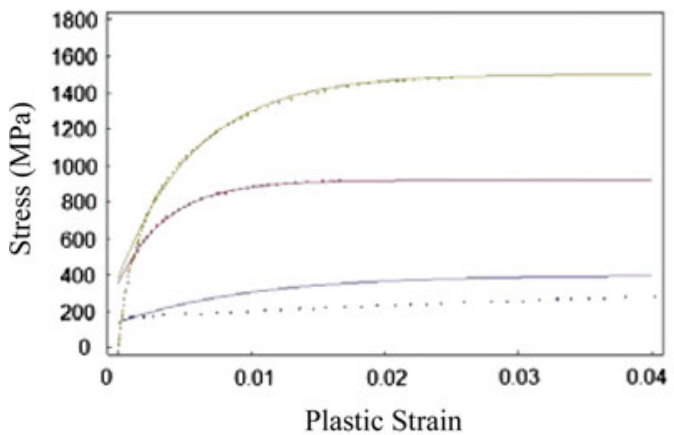

$\left(\mathbf{a}^{*}\right)$

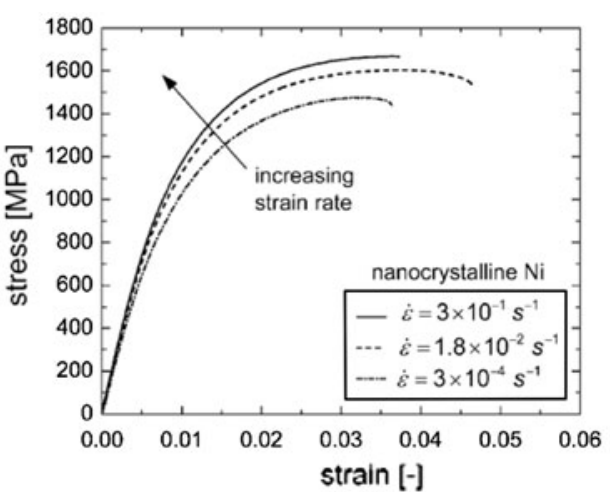

(b)

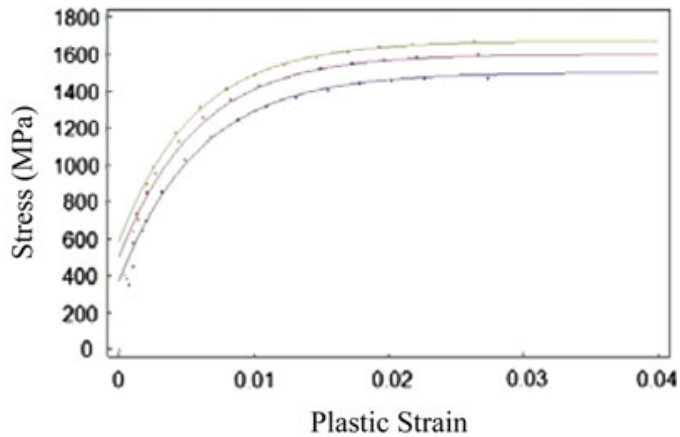

(b*)

Fig. 11-Simultaneous effect of grain size and strain rate on the $\sigma-\varepsilon$ relation for nc-Cu: $(a)$ and $(b)$ Experimental graphs ${ }^{[32]} ;\left(a^{*}\right)$ and $\left(b^{*}\right)$ Model predictions. 
functions $\left(\sigma_{s}, \sigma_{f}, h\right)$ and the parameter values used for these fits are shown subsequently:

$$
\begin{gathered}
\sigma_{f}=\left(\sigma_{f}^{0}+\frac{k_{1}}{\sqrt{d}}-\frac{k_{2}}{d}\right)\left(1+m_{f} \ln \left(\frac{\dot{\varepsilon}_{p}}{\dot{\varepsilon}_{p}^{0}}\right)\right) \\
\sigma_{s}=\left(\sigma_{s}^{0}+\frac{k_{3}}{\sqrt{d}}-\frac{k_{4}}{d}\right)\left(1+m_{s} \ln \left(\frac{\dot{\varepsilon}_{p}}{\dot{\varepsilon}_{p}^{0}}\right)\right) \\
h=h_{0}+\frac{k_{5}}{\sqrt{d}}-\frac{k_{6}}{d}
\end{gathered}
$$

\begin{tabular}{|c|c|c|c|c|c|c|c|}
\hline \multicolumn{3}{|c|}{$\sigma_{f}^{0}$} & \multicolumn{3}{|c|}{$\sigma_{s}^{0}$} & \multicolumn{2}{|c|}{$h_{0}$} \\
\hline \multicolumn{3}{|c|}{$70 \mathrm{MPa}$} & \multicolumn{3}{|c|}{$265 \mathrm{MPa}$} & \multicolumn{2}{|c|}{$3 \mathrm{GPa}$} \\
\hline$k_{1}$ & $k_{2}$ & $m_{f}$ & $k_{3}$ & $k_{4}$ & $m_{s}$ & $k_{5}$ & $k_{6}$ \\
\hline 386 & 1634 & 0.04 & 437 & 1207 & 0.01 & 43,460 & 153,68 \\
\hline
\end{tabular}

with the units for $k$ 's expressed in $\mathrm{kPa} \sqrt{\mathrm{m}}$. The $d^{-1 / 2}$ and $d^{-1}$ dependence of $\left(\sigma_{s}, \sigma_{f}, h\right)$ is in accordance with the discussion provided at the end of Section III-B-1.

\section{ACKNOWLEDGMENTS}

Discussions with K.E. Aifantis and X. Zhang are acknowledged, as well as the support of the ERC grant of the former to the latter for carrying out some of the curve fittings. The same holds for my colleague A. Konstantinidis and doctoral student J. Konstantopoulos for going through portions of the manuscript.

\section{REFERENCES}

1. E.C. Aifantis: Mech. Res. Comm., 1978, vol. 5, pp. 139-45.

2. E.C. Aifantis: J. Eng. Mater. Technol., 1984, vol. 106, pp. 326-30.

3. E.C. Aifantis: Int. J. Plast., 1987, vol. 3, pp. 211-47.

4. E.C. Aifantis: J. Mech. Behav. Mater., 1994, vol. 5, pp. 355-75.

5. J. Horvath, R. Birringer, and H. Gleiter: Solid State Comm., 1987, vol. 62 , pp. 319-22.
6. B.S. Bokhstein and L.I. Trusov: Def. Diffus. Forum, 1993, vols. 9598, pp. 445-48.

7. E.C. Aifantis: J. Appl. Phys., 1979, vol. 50, pp. 1334-38.

8. E.C. Aifantis: Acta Metall., 1979, vol. 27, pp. 683-91.

9. T.D. Shen, C.C. Koch, T.Y. Tsui, and G.M. Pharr: J. Mater. Res., 1995, vol. 10, pp. 2892-96.

10. J.E. Carsley, J. Ning, W.W. Milligan, S.A. Hackney, and E.C. Aifantis: Nanostr. Mater., 1995, vol. 5, pp. 441-48.

11. D.A. Konstantinidis and E.C. Aifantis: Nanostr. Mater., 1998, vol. 10 , pp. 1111-18.

12. M.A. Meyers, A. Mishra, and D.J. Benson: Progr. Mater. Sci., 2006, vol. 51, pp. 427-556.

13. C.S. Pande and K.P. Cooper: Progr. Mater. Sci., 2009, vol. 54, pp. 689-706.

14. G. Saada and G. Dirras: in Dislocations in Solids, J.P. Hirth and L. Kubin, eds., 2009, pp. 199-248.

15. K.E. Aifantis and A.A. Konstantinidis: Mater. Sci. Eng. A, 2009, vol. 503, pp. 198-201.

16. K.E. Aifantis and A.A. Konstantinidis: Mater. Sci. Eng. B, 2009, vol. 163 , pp. 139-44.

17. C.A. Schuh, T.C. Hugnagel, and U. Ramamurty: Acta Mater., 2007, vol. 55, pp. 4067-4109.

18. X.H. Zhu, J.E. Carsley, W.W. Milligan, and E.C. Aifantis: Scripta Mater., 1997, vol. 36, pp. 721-26.

19. J.E. Carsley, W.W. Milligan, X.H. Zhu, and E.C. Aifantis: Scripta Mater., 1997, vol. 36, pp. 727-32.

20. E.C. Aifantis and J.M. Hill: Q. J. Mech. Appl. Math., 1980, vol. 33, pp. 1-21.

21. J.M. Hill and E.C. Aifantis: Q. J. Mech. Appl. Math., 1980, vol. 33 , pp. 23-41.

22. D.A. Konstantinidis and E.C. Aifantis: Scripta Mater., 1999, vol. 40 , pp. $1235-41$.

23. E.C. Aifantis: Mech. Mater., 2003, vol. 35, pp. 259-80.

24. J. Kioseoglou, G.P. Dimitrakopulos, P. Komninou, T. Karakostas, I. Konstantopoulos, M. Avlonitis, and E.C. Aifantis: Phys. Status Solidi A, 2006, vol. 203, pp. 2161-66.

25. E.C. Aifantis: Mater. Sci. Eng. A, 2009, vol. 503, pp. 190-97.

26. E.C. Aifantis: Dislocations 2008, IOP Conf. Series, W. Cai, K. Edagawa, and A.H.W. Ngan, eds.: Mater. Sci. Eng., 2009, vol. 3, pp. 012026/1-012026/10.

27. K.E. Aifantis and J.R. Willis: J. Mech. Phys. Solids., 2005, vol. 53, pp. $1047-70$

28. K.E. Aifantis and A.H.W. Ngan: Mater. Sci. Eng. A, 2007, vol. 459 , pp. 251-61.

29. A.S. Khan, B. Farrokh, and L. Takacs: J. Mater. Sci., 2008, vol. 43 , pp. $3305-13$.

30. J. Schiøtz, T. Vegge, F.D. di Tolla, and K.W. Jacobsen: Phys. Rev. $B, 1999$, vol. 60, pp. 11971-83.

31. X. Zhang, A.E. Romanov, and E.C. Aifantis: Mater. Sci. Forum, 2011, vols. 667-669, pp. 991-96; also K.E. Aifantis and X. Zhang: ERC/LMM-AUT Report, Aristotle University, Thessaloniki GR, 2010.

32. R. Schwaiger, B. Moser, M. Dao, N. Chollacoop, and S. Suresh: Acta Mater., 2003, vol. 51, pp. 5159-72. 